\title{
Favorable Results of Patients with pT1a, b, c, Lymph Node-negative Early Breast Cancer in the Long-interval \\ N Bulut ${ }^{1}$, K Altundag ${ }^{1}$
}

\begin{abstract}
Background: The clinical characteristics of patients who had lymph node-negative early breast cancer were assessed.

Methods: We assessed a total of 579 patients in the study, including 74 patients $(12.8 \%)$ with T1a, 119 patients (20.7\%) and 367 patients (63.7\%) with T1c. Patients with T1 tumors were further classified into 3 groups according to hormone receptor (HR) and HER-2 status.

Results: In patients with T1a, the disease-free survival (DFS) rate was $98.4 \%$ at 2 years $(\mathrm{p}=0.000)$. In subgroup analysis of early breast cancers , 10-year-DFS rates of the patients in HR(+)/HER2(-), HR(- ) /HER2(+) and HR(-)/HER2(-) subgroups were no significant difference $(p=0.917)$.
\end{abstract}

Conclusion: The T1a group had a worse prognosis than T1b and T1c groups in second years $(\mathrm{p}=0.000)$, but there was no statistically important difference between subgroups $(\mathrm{p}=0.917)$. Although there are differences in patients and tumor features, the prognosis of patients with T1a, b, cN0M0 breast cancer is excellent.

From: Department of Medical Oncology, Hacettepe University Institute of Oncology ${ }^{1}$, Ankara, Turkey, Kadri Altundag.

Correspondence: Dr N Bulut, Hacettepe University Institute of Oncology, Department of Medical Oncology, Sihhiye, 06100, Ankara, Turkey, Fax: +90 3123092905 e-mail: ferlut@hotmail.com 


\section{INTRODUCTION}

Early breast cancers comprise of almost $50 \%$ of all breast cancers. In some cases, only $10 \%$ of all breast cancers were categorized in the early breast cancer category (1). Patients with lymph node-negative invasive breast carcinomas $<$ or $=1 \mathrm{~cm}$ in size have a low incidence of recurrence. These tumors are most often found during a mammographic examination of breast tissue.

Most node-negative breast cancers with the primary $\leq 2 \mathrm{~cm}(\mathrm{~T} 1 \mathrm{a}, \mathrm{b}, \mathrm{c}$ N0M0) do not recur within $\geq 20$ years of follow up when treated with a mastectomy, lumpectomy, or a lumpectomy followed by radiation therapy $(1,2)$.

It has been reported that the prognosis of a small-sized with node-negative breast cancer is excellent with complete surgery resection of the primary tumor, even without systemic adjuvant therapy (3). Since the subgroups of patients who were diagnosed with small, nodenegative breast cancer tumors have an increased risk of recurrence, these patients are likely to benefit from adjuvant therapy. Especially, young patients under 35 years old with a poor prognosis. The biological factors of a high-grade tumor, HER2/neu status and high Ki-67, tumor size, nodal involvement, lymphovascular invasion were related to a high recurrence or high mortality rate $(3,4)$.

In this study, the overall prognosis of lymph node-negative invasive ductal carcinoma (IDC) of the breast that were $\leq 2 \mathrm{~cm}$ in size was investigated retrospectively. Prognostic and predictive factors in subgroups of patients were evaluated, and also some of the patients who would be candidates for systemic adjuvant and hormonal therapies were determined. 


\section{PATIENTS AND METHODS}

The women who were diagnosed with breast cancer from 1981 to 2013 in our clinic were included in this analysis. Patient demographics were procured from charts. Tumors were marked according to the modified Bloom-Richardson scoring system and staged according to the TNM criteria. The data on ER, PR, and HER2/neu were procured through standard clinical testing, using immune-histochemistry (IHC) for ER and PR and the HerceptTest for HER2/neu. For ER and PR, receptor positivity was based on more than 5\% of cells testing positive. The IHC was scored on a qualitative scale from 0 to $3+$, based on an interpretation of the staining intensity, with 0 and $1+$ categorized as negative (incomplete membrane staining in $10 \%$ of the tumor cells), $2+$ as borderline, and $3+$ as positive (strong and complete membrane staining in $>10 \%$ of cells).

Tumors that scored $2+$ were further analyzed for HER2 amplification by means of fluorescence in situ hybridization (FISH). T1a, b, c N0M0 are stage 1 breast cancers that are categorized, when the T1a is no more than $0.5 \mathrm{~cm}$, T1b is 0.6 to $1.0 \mathrm{~cm}$ and $\mathrm{T} 1 \mathrm{c}$ is more than $1 \mathrm{~cm}$ but less than $2 \mathrm{~cm}$.

Traditionally, the status of axilla has been evaluated by a standard axillary dissection with a total mastectomy in which level I and II lymph nodes were removed. A sentinel lymph node biopsy was performed to mark the stage of the axilla by using a blue dye method. All of the lumpectomy-treated patients received radiation therapy (RT). A total of $38(6.6 \%)$ women were treated with surgery alone; $216(37.4 \%)$ women received chemotherapy; 322 (55.9\%) received only hormonal therapy (tamoxifen and/or aromatase inhibitor); and 116 (20.1\%) received chemotherapy followed by hormonal therapy. 


\section{Statistical analysis}

The differences in clinical and biological characteristics between T-stage subgroups were evaluated by a chi-square test. The recurrence-free survival (RFS) curves were constructed using the Kaplan-Meier method, and the log-rank test was used for a comparison of the survival curves between subgroups according to prognostic factors. Also the five-year cumulative survival rate and the association between the probability of death or recurrence and the clinical and biologic features were compared. A significance level of 0.05 was used for covariate entry. All statistical analyses were performed using SPSS for Windows software, version 15.0 .

\section{RESULTS}

A total of 579 patients were involved in the study. Median age was 54 (range: 27 to 86 years) amongst the patients with T1a, T1b and T1c tumors. Seventy-four patients (12.8\%) had T1a tumors, 119 patients $(20.7 \%)$ had T1b, 367 patients $(63.7 \%)$ had T1c tumors.

Seventy-five patients (14.6\%) had HER2+ tumors; $81.7 \%$ had a hormone receptor positive disease; $12.4 \%$ had a triple-negative disease. Patients with T1 were further classified into HR+/HER2-, HR+/HER2+, HR-/HER2- and HR-/HER2+ subgroups (table).

Two hundred and eighty patients (48.8\%) had a mastectomy and breast-conserving surgery (BCS) was performed on 297 women (51.9\%). Among patients who received BCS, 297 patients $(51.9 \%)$ were treated with adjuvant radiotherapy after surgical resection. One hundred and seventy-one patients $(29.6 \%)$ received systemic adjuvant therapy. We treated these patients with a mixed chemotherapy treatment consisting of cyclophosphamide, methotrexate, fluorouracil and anthracycline-based regimen. Among patients with hormonereceptor positive disease, $439(76.0 \%)$ received adjuvant endocrine therapy. Seventy-three 
patients $(14.1 \%)$ were HER2(+) and 46 patients $(8.0 \%)$ received adjuvant trastuzumab who clinically at high risk.

Thirty-seven patients $(6.4 \%)$ who received hormonal therapy were switched from tamoxifen to aromatase inhibitors after two or three years. We followed 38 patients (6.6\%) without any treatment. Median follow-up was 4.2 years. In patients with T1a, b, c, the disease freesurvival (DFS) rate was $98.4,100$ and $100 \%$ in the second year, respectively $(\mathrm{p}=0.000)$. At year 10, DFS rates of T1a, T1b, and T1c patients were 95.7, 100 and 100\%, respectively $(\mathrm{p}=0.000)$. There was difference at year 20 in the DFS rate between T1b and T1c patients [100\% for T1b, $80 \%$ for T1c $9(\mathrm{p}=0.000)]$ (Figure 1). Median overall survival rates could not be procured. In the subgroup analysis of T1; two and 10 years DFS rates were $99.7 ; 93.4 \%$ in HR+/HER2-; $100 \%$ in both HR-/HER2-, 100\% in both HR-/HER2+ patients, respectively. There was no significant difference between HR+/HER2-, HR-/HER2- and HR-/HER2+ subgroups $(\mathrm{p}=0.917)$ (Figure 2).

\section{DISCUSSION}

It is standard practice to use adjuvant systemic therapy for all patients with a lymph nodepositive disease (5). There are many node-negative women who would benefit from adjuvant systemic therapy. A prognostic factor is seen as a result of surgery is to be disease free and overall survival of the systemic untreatable disease. Some factors, such as hormone receptor status, HER2/neu overexpression, grade, vascular invasion, tumor size, p53, mitotic index are prognostic and predictive (2). These factors predict the risk of recurrence and are used for the selection of patients for adjuvant systemic therapy $(1,6)$. 
Retrospective studies showed that T1a, b breast cancers were a small population. Patients with a grade 3 tumor had less than a 10-year DFS rate compared to grade 1 and 2 patients (3). Age, tumor size, lymphovascular invasion and estrogen receptor negativity were commonly found to be poor prognostic factors (2). Also HER2+ disease is a recurrence risk factor in node-negative tumors (3). In our study, there was no difference in the DFS rates between the grade 1,2 or 3 in patients with $\mathrm{T} 1$ breast cancer $(\mathrm{p}=0.130)$. Also, statistically there was no difference in the DFS rates in patients between $\leq 35$ years old and $\geq 35$ years old and HRpatients ( $\mathrm{p}=0.216$ and $\mathrm{p}=0.917$, respectively). In HR+/HER2-, HR-/HER2- and HR-/HER2+ subgroups had a good prognosis within 4.2 years $(\mathrm{p}=0.917)$.

The purpose of adjuvant chemotherapy is to eradicate distant micrometastatic diseases. For node-negative patients, tumor size is the most powerful prognostic factor and is routinely used in making adjuvant treatment decisions.

In general, patients with a tumor size of $>1-2 \mathrm{~cm}$ warrant consideration of adjuvant therapy since they may have a relapse risk of $20-30 \%(2,4)$. In this study, a high lymphovascular invasion (LVI) in T1c tumors $(\mathrm{p}=0.002)$ was defined. The presence of LVI had an effect with respect to the risk of recurrence $(\mathrm{p}=0.033)$. Thirty-six $(6.3 \%)$ of 367 patients with $\mathrm{T} 1 \mathrm{c}$ tumors developed systemic relapse.

It has been demonstrated in our study that adjuvant hormonal therapy and hormonal therapy plus chemotherapy decreased the risk of recurrence and death from breast cancer.

$\mathrm{ER}+$ tumors in patients who were treated with chemotherapy and tamoxifen and/or aromatase inhibitors $(26.5 \%)$, regardless of the type of surgery. The rates of relapse and metastases were low. Especially, $\mathrm{CMF}$ and $\mathrm{AC}$ regimens have reduced recurrence risk in the late interval $(4,7)$. Therefore, we cannot show if the pattern of recurrence reflects the natural history of specific subgroups or a response to the adjuvant therapies received. 
Some studies showed that HER2/neu amplification related to worsened DFS rates. But anthracyline-based chemotherapy regimens improved the risk of recurrence (8). Similar to our study, Kwon J.H. et al. found a good short-term prognosis in lymph node-negative breast cancers (3). In contrast, a recent assessment of 194 node-negative pT1a, b breast cancer, 58\% treated with adjuvant chemotherapy, showed an excellent five-year outcome irrespective of chemotherapy (9).Very few women with small node-negative tumors (T1a, b N0) were found in large adjuvant trastuzumab studies. Retrospective studies showed poor outcomes for patients with small HER2+ tumors than HER2- disease, in those that suggested adjuvant trastuzumab therapy (10). In our study, 73 patients had HER2+ T1N0 tumors and 46 received adjuvant trastuzumab.

However, an increased mortality rate was not seen in women with HER2+ that were not treated with adjuvant trastuzumab during the five years after diagnosis at the end of followup. A significant improvement in survival rates of women who were treated with adjuvant trastuzumab could not be performed, but a direction for future research was represented. As a result, the combination of hormonal therapy and trastuzumab may be particularly beneficial for $\mathrm{ER}+$ patients.

In our results, the DFS rate was $98.4,100$, and $100 \%$ in the second year, respectively $(\mathrm{p}=0.000)$ for patients with T1a, b, c. The 11-year cumulative survival rate was $93.4 \%$ in HR+/HER2-, 100\% in HR-/HER2+ and HR-/HER2- in patients ( $\mathrm{p}=0.917)$. Forty-eight ( $8.3 \%)$ of the patients with T1a tumor received hormonal therapy. Sixteen (2.8\%) T1a tumor patients were untreated after surgery. Forty-six $(8.0 \%)$ women were treated with modified radical mastectomy and $28(4.9 \%)$ women had breast conservative surgery (BCS). All the BCS-treated patients received radiation therapy. In a Kennecke H. et al. study, a subgroup of women with early ER+ breast cancer were identified to be at increased risk of recurrence within 2-5 years of diagnosis despite treatment with tamoxifen, just as in our study $(11,12)$. 
Previous studies demonstrated that a high grade was prognostic of poorer outcomes among women with ER+ early breast cancers $(6,11)$. Low ER levels were predictive of poorer outcomes among tamoxifen-treated patients (11). The second recurrence peak is higher for ER+ and lower for ER-tumors (6).

Ipsilateral breast cancer recurrence may also be caused by tumor cells lodged within the breast in which adjuvant RT is administered. RT causes both a conventional short-term tumoricidal effect and the so called "tumor bed effect", in which the radiation-induced vascular damage of the surrounding tissue more indirectly impairs tumor growth (6). The different recurrence peak timing is related to a differential effect of RT on ER+ and ERnegative tumor cells. This hypothesis is that ER- tumor cells display higher radiosensitivity than ER+ tumor cells (13). The risk of a recurrence was greater in women who had tumors of $\geq 1 \mathrm{~cm}$ in size regardless of ER status (14).

Women with grade 3 tumors had a $\geq 5 \%$ annual risk of recurrence within the first five years after a diagnosis with a peak annual risk of $7.6 \%$ during the second year after diagnosis. The annual recurrence risk of for women with grade 1 or 2 tumors was relatively consistent, at approximately $2 \%$ per year beyond the first year after diagnosis. Women who had lymph node-negative tumors had a persistent an approximate $2 \%$ per year recurrence risk (11).

In our study, the difference of DFS rates might be caused by the gene signature of patients with T1a in the second year. In genetic analysis, four chromosomal regions associated with poor prognosis included: 17q12, 17q21.33-q25.1, 8p11.2 and 8q24.3 in ER+HER2- breast cancers. This situation is associated with relative resistance to hormonal therapy. These amplification regions were strong predictors of early recurrence of ER+ breast cancers (12).

In estrogen-dependent breast tumors, aromatase over-expression could cause cell proliferation. The estrogen, EGF/EGFR and c-kit pathways can also control snail and slug expression patterns. In breast carcinomas, snail transcription factors have been linked to 
tumor progression and invasiveness. Epithelial mesenchymal transition (EMT) plays a significant role in the development of metastases. One of the hallmarks of EMT is the loss of E-cadherin and a gain of $\mathrm{N}$-cadherin expression, which are regulated by transcription factors, such as snail, slug and twist. The level of snail $(\mathrm{p}=0.014)$ and twist $(\mathrm{p}=0.006)$ expressions were associated with a worsened patient relapse-free period, specifically in patients with ERpositive tumors $(\mathrm{p}=0.039)(15)$. A pAkt expression related to a poor grade and positive nodal status in early breast cancer. Also, pAkt and pErk1/2 led to a chemo-resistance of breast cancer (16).

An excellent prognosis of lymph-node negative IDC of the breast of $2 \mathrm{~cm}$ or less was determined in our study. Among the 576 patients, in a survival analysis only $7.3 \%$ of the patients relapsed and $0.6 \%$ died during follow-up. Median overall survival rates could not be assessed. Patients might have had a good prognosis because of combine chemotherapy regimens and a non-aggressive biologic phenotype. The J. M. You et al. study showed a good prognosis in all molecular sub-types (17). These studies included a short follow-up for early breast cancer in patients ( approximately five years) $(3,11)$. In our patients who received radiation treatment after breast-conserving therapy, only surgery and chemotherapy and/or hormonal therapy were similar in disease-free survival rates.

In conclusion, all $\mathrm{T} 1$ breast cancers showed to have excellent long-term outcome regardless of initial treatment. Recurrences and deaths are known to happen, even if less frequently, in small, node-negative breast cancer patients, and the decision on adjuvant treatments remains controversial. The introduction of molecular sub-types and gene signatures improve the early breast cancer treatment, and individual institutes have to find their policy based on their histopathological information and the availability of gene signatures. 


\section{ACKNOWLEDGEMENTS}

This study's data was procured from Medical Oncology Institution of Hacettepe University. We did not receive financial support for this project. 


\section{REFERENCES}

1. Joensuu H., Isola J., Lundin M., Salminen T., Holli K., Kataja V., et al . Amplification of erB2 and erbB2 expression are superior to estrogen receptor status as risk factors for distant recurrence in pT1N0M0 breast cancer: A Nationwide population-based study. Clin Cancer Res, 2009; 9(3):923-30.

2. Cranfrocca M., Goldstein LJ . Prognostic and predictive factors in early-stage breast cancer. The Oncol 2004;9(6): 606-16.

3. Kwon JH., Kim YJ., Lee KW., Oh DY., Park SY., Kim JH., et al . Triple negativity and young age as prognostic factors in lymph node-negative invasive ductal carcinoma of $1 \mathrm{~cm}$ or less. BMC Cancer 2010; 15(10): 557. doi: 10.1186/1471-2407$10-557$.

4. Kennecke H., Yerushalmi R., Woods R., Cheang MC., Voduc D., Speers CH., et al. Metastatic behavior of breast cancer subtypes. J Clin Oncol 2010; 28 (20):3271-7.

5. Mirza AN., Mirza NQ., Vlastos G., Singletary SE. Prognostic factors in nodenegative breast cancer. Ann of Surg 2002; 235 (1) : 10-26.

6. Chia SK., Speers CH., Bryce CJ., Hayes MM., Olivotto IA. Ten-year outcomes in a population-based cohort of node-negative, lymphatic, and vascular invasion-negative early breast cancers without adjuvant systemic therapies. J Clin Oncol 2004; 22(9): $1630-7$.

7. Dignam JJ., Dukic V., Anderson SJ., Mamounas EP., Wickerham DL., Wolmark N., et al . Hazard of recurrence and adjuvant treatment effects over time in lymph nodenegative breast cancer.Breast Cancer Res Treat 2009; 116(3): 595-602.

8. Mahyari HM, Khosravi A., Mahyari ZM., Monfared EZ., Khosravi N. Overexpression of HER2/neu as a prognostic value in Iranian women with early stage 
breast cancer; A single Institute Study. Iran Red Crescent Med J 2014; 16(11). doi:10.5812/ircmj.16005.eCollection 2014.

9. Ho AY., Gupta G., King TA., Perez CA., Patil SM., Rogers KH., et al . Favorable prognosis in patients with $\mathrm{T} 1 \mathrm{a} / \mathrm{T} 1 \mathrm{bN} 0$ triple-negative breast cancers treated with multimodality therapy. Cancer 2012; 118 (20):4944-52.

10. Seal MD., Speers CH., O’Reilly S., Gelmon KA., Ellard SL.,Chia SK. Outcomes of women with early-stage breast cancer receiving adjuvant trastuzumab. Curr Oncol 2012;19 (4): 197-01.

11. Kennecke H., McArthur H., Olivotto IA., Speers C., Bajdik C., Chia SK., et al. Risk of early recurrence among postmenopausal women with estrogen receptor-positive early breast cancer treated with adjuvant tamoxifen. Cancer 2008; 112 (7):1437-44.

12. Bilal E., Vassallo K., Toppmeyer D., Barnard N., Rye IH., Almendro V., et al. Amplified loci on chromosomes 8 and 17 predict early relapse in ER-positive breast cancers. Plos One 2012;7 (6):1-10.

13. Demicheli R., Ardoino I., Boracchi P., Coradini D., Agresti R., Ferraris C.,et al. Recurrence and mortality according to Estrogen receptor status for breast cancer patients undergoing conservative surgery. Ipsilateral breast tumour recurrence dynamics provides clues for tumour biology within the residual breast. BMC Cancer 2010; 30(10):656.

14. Fisher B., Dignam J., Tan-Chiu E., Anderson S., Fisher ER., Wittliff JL., et al. Prognosis and treatment of patients with breast tumors of one centimeter or less and negative axillary lymph nodes. J Natl Cancer Inst 2001; 93 (2):112-20.

15. Van Nes JG., de Kruijf EM., Putter H., Faratian D., Munro A., Campbell F.,et al. Coexpression of Snail and Twist determines prognosis in estrogen receptor-positive early breast cancer patients. Breast Cancer Res Treat 2012;133(1):49-59. 
16. Liu W, Zhang L., Shı J., Liu Y., Zhou L., Hou K. et al. Clinical significance of pAkt and pErk $1 / 2$ expression in early stage breast cancer patients treated with anthracycline-based adjuvant chemotherapy. Oncol Lett 2005;9 (4):1707-14.

17. You JM, Kim YG., Moon HG., Nam SJ., Lee JW., Lim W. et al . Survival improvement in Korean breast cancer patients due to the increases in early-stage cancers and hormone receptor positive/HER 2 negative suptypes: A nationwide registry -based study. J Breast Cancer 2015;18(1): 8-15. 
Table: Baseline characteristics of all patients according to $\mathrm{T}$ stage

\begin{tabular}{|c|c|c|c|c|c|c|}
\hline & & $\begin{array}{l}\text { All patients } \\
(N=576)\end{array}$ & $\begin{array}{l}\text { T1a } \\
(N=74)\end{array}$ & $\begin{array}{l}\text { T1b } \\
(N=119)\end{array}$ & $\begin{array}{l}\text { T1c } \\
(N=367)\end{array}$ & P value \\
\hline \multirow[t]{3}{*}{ Grad } & 1 & $127(25.2)$ & $18(3.6)$ & $35(6.9)$ & $74(14.7)$ & 0.130 \\
\hline & 2 & $224(44.5)$ & $24(4.8)$ & $43(8.5)$ & $157(31.2)$ & \\
\hline & 3 & $140(27.8)$ & $13(2.6)$ & $31(6.2)$ & 96(19) & \\
\hline \multirow[t]{2}{*}{ ER } & negative & $105(18.6)$ & $19(3.4)$ & $21(3.8)$ & $64(11.4)$ & 0.271 \\
\hline & positive & $440(78.6)$ & $49(8.8)$ & $96(17.1)$ & $295(52.7)$ & \\
\hline \multirow[t]{2}{*}{ PR } & negative & $127(22.9)$ & $19(3.4)$ & $26(4.7)$ & $82(14.8)$ & 0.307 \\
\hline & positive & $410(74.2)$ & $48(8.7)$ & $89(16.1)$ & $273(49.4)$ & \\
\hline \multirow[t]{2}{*}{ HER } & 2 negative & $421(81.8)$ & $37(7.2)$ & $90(17.5)$ & $294(57.1)$ & 0.177 \\
\hline & positive & $73(14.1)$ & $14(2.7)$ & $16(3.1)$ & $43(8.3)$ & \\
\hline \multicolumn{7}{|c|}{ HR/HER2 } \\
\hline \multicolumn{2}{|c|}{$\mathrm{HR}+/ \mathrm{HER} 2+$} & $44(8.7)$ & $6(1.2)$ & $5(1.0)$ & $33(6.5)$ & 0.002 \\
\hline \multicolumn{2}{|c|}{$\mathrm{HR}+/ \mathrm{HER} 2-$} & $360(71.1)$ & $32(6.3)$ & $83(16.4)$ & $245(48.4)$ & \\
\hline \multicolumn{2}{|c|}{ HR-/HER2- } & $62(12.3)$ & $9(1.8)$ & $8(1.6)$ & $45(8.9)$ & \\
\hline \multicolumn{2}{|c|}{ HR-/HER2+ } & $26(5.2)$ & $7(1.4)$ & $11(2.2)$ & $8(1.6)$ & \\
\hline \multicolumn{7}{|c|}{$\begin{array}{l}\text { Lymphovascular } \\
\text { invasion (LVI) }\end{array}$} \\
\hline & No & $61(35.9)$ & $10(7.5)$ & $17(2.8)$ & $34(25.6)$ & \\
\hline \multicolumn{7}{|c|}{ Menopausal } \\
\hline & Peri & $48(8.3)$ & $6(1.0)$ & $8(1.4)$ & $34(5.9)$ & \\
\hline & Post & $268(46.6)$ & $30(5.2)$ & $64(11.1)$ & $174(30.3)$ & \\
\hline
\end{tabular}


Fig.1: Recurrence -free survival according to T1 size.

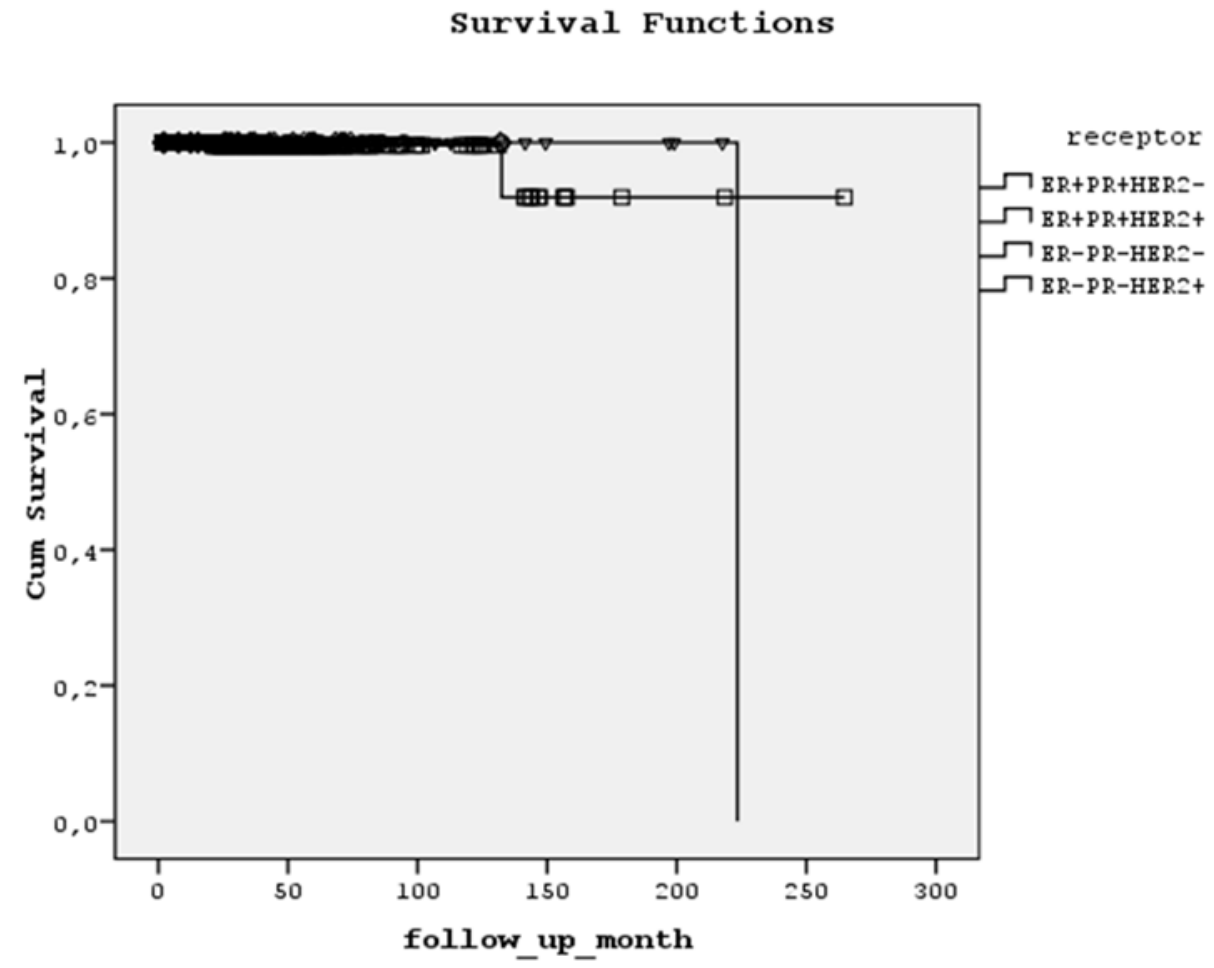

Fig.2: Analysis of disease-free survival of four breast cancer subtypes by log-rank test 